Pure and Applied Mathematics Quarterly

Volume 3, Number 2

(Special Issue: In honor of

Leon Simon, Part 1 of 2)

417-449, 2007

\title{
Minkowski Type Problems for Convex Hypersurfaces in the Sphere
}

\author{
Claus Gerhardt
}

Dedicated to Leon Simon on the occasion of his 60th birthday

\begin{abstract}
We consider the problem $F=f(\nu)$ for strictly convex, closed hypersurfaces in $S^{n+1}$ and solve it for curvature functions $F$ the inverses of which are of class $(K)$.
\end{abstract}

\section{Contents}

0. Introduction 418

1. Notations and definitions 420

2. Polar sets 422

3. Curvature estimates 432

4. Lower order bounds 434

5. A uniqueness result 439

6. Existence of a solution 440

7. Proof of Theorem $0.4 \quad 447$

References $\quad 448$

Received September 10, 2005.

2000 Mathematics Subject Classication. 35J60, 53C21, 53C44, 53C50, 58J05.

Key words and phrases: Minkowski problem, hypersurfaces of prescribed curvature, Weingarten hypersurfaces. 


\section{Introduction}

In the classical Minkowski problem in $\mathbb{R}^{n+1}$ one wants to find a strictly convex closed hypersurface $M \subset \mathbb{R}^{n+1}$ such that its Gauß curvature $K$ equals a given function $f$ defined in the normal space of $M$ or equivalently defined on $S^{n}$

$$
K_{\left.\right|_{M}}=f(\nu) .
$$

The problem has been partially solved by Minkowski [13], Alexandrov [1], Lewy [11], Nirenberg [14], and Pogorelov [15], and in full generality by Cheng and Yau $[2]$.

Instead of prescribing the Gaussian curvature other curvature functions $F$ can be considered, i.e., one studies the problem

$$
F_{\left.\right|_{M}}=f(\nu) \text {. }
$$

If $F$ is one of the symmetric polynomials $H_{k}, 1 \leq k \leq n$, this problem has recently been solved by Guan and Guan [9]. They proved that (0.2) has a solution, if $f$ is invariant with respect to a fixed point free group of isometries of $S^{n}$.

In this paper we consider the problem (0.2) for strictly convex hypersurfaces $M \subset S^{n+1}$ and for curvature functions $F$ the inverses of which are of class $(K)$, see Section 1 or $\left[8\right.$, Definition 1.3]. These $F$ include all $H_{k}, 1 \leq k \leq n,|A|^{2}$, and also any symmetric, convex curvature function homogeneous of degree 1 , cf. [7, Lemma 1.6].

We shall show in Section 2 that for any closed strictly convex hypersurface $M \subset S^{n+1}$ there exists a Gauß map

$$
x \in M \rightarrow \tilde{x} \in M^{*},
$$

where $M^{*}$ is the polar set of $M . M^{*}$ is also strictly convex, as smooth as $M$, and the Gauß map is a diffeomorphism.

If we consider $M$ as an embedding in $\mathbb{R}^{n+2}$ of codimension 2 , so that the tangent spaces $T_{x}(M)$ and $T_{x}\left(S^{n+1}\right)$ can be identified with subspaces of $T_{x}\left(\mathbb{R}^{n+2}\right)$, then the image of the point $x$ under the Gauß map is exactly the normal vector $\nu \in T_{x}\left(S^{n+1}\right)$

$$
\tilde{x}=\nu \in T_{x}\left(S^{n+1}\right) \subset T_{x}\left(\mathbb{R}^{n+2}\right) .
$$

Thus, the equation (0.2) can also be written in the form

$$
F_{\left.\right|_{M}}=f(\tilde{x}) \quad \forall x \in M,
$$

where $f$ is given as a function defined in $S^{n+1}$.

We shall also prove that (0.5) has a dual problem, namely,

$$
\tilde{F}_{\left.\right|_{M^{*}}}=f^{-1}(\tilde{x}) \quad \forall \tilde{x} \in M^{*},
$$


where $\tilde{F}$ is the inverse of $F$

$$
\tilde{F}\left(\kappa_{i}\right)=\frac{1}{F\left(\kappa_{i}^{-1}\right)}
$$

In the dual problem the curvature is not prescribed by a function defined in the normal space, but by a function defined on the hypersurface.

Both problems are equivalent, solving one also leads to a solution of the dual one; notice also that

$$
M^{* *}=M \quad \wedge \quad \tilde{\tilde{x}}=x .
$$

To find a solution we either impose some symmetry requirement with respect to a group of isometries or we assume the existence of barriers.

0.1. Assumption. (i) Let $G \subset O(n+2)$ be a group of orthogonal transformations with a common fixed point $x_{0} \in S^{n+1}$ and assume that the induced group of isometries in $S^{n}$, i.e., the equator of the hemisphere with center in $x_{0}$, is fixed point free.

(ii) Let $0<f \in C^{5}\left(S^{n+1}\right)$ be invariant with respect to the group $G$, i.e.,

$$
f(A x)=f(x) \quad \forall x \in S^{n+1}, \forall A \in G .
$$

Then we shall prove

0.2. Theorem. Let $F \in C^{5}\left(\Gamma_{+}\right)$be a symmetric, positively homogeneous and monotone curvature function such that its inverse $\tilde{F}$ is of class $(K)$, then the dual problems

$$
F_{\left.\right|_{M}}=f(\tilde{x})
$$

and

$$
\tilde{F}_{\left.\right|_{M^{*}}}=f^{-1}(\tilde{x})
$$

have strictly convex solutions $M$ resp. $M^{*}$ of class $C^{4, \alpha}, 0<\alpha<1$, such that the hypersurfaces $M$ resp. $M^{*}$ are invariant with respect to the group $G$. Furthermore, $-x_{0}$ is an interior point of the convex body $\hat{M}$ and $x_{0}$ an interior point of the convex body $\hat{M}^{*}$ of $M^{*}$. The convex bodies $\hat{M}, \hat{M}^{*}$ are strictly contained in the corresponding open hemispheres $\mathcal{H}\left(-x_{0}\right)$ resp. $\mathcal{H}\left(x_{0}\right)$.

Instead of imposing some symmetry assumption, a barrier condition will also work.

0.3. Assumption. Let $M_{i}, i=1,2$, be strictly convex hypersurfaces of class $C^{6, \alpha}$ contained in an open hemisphere $\mathcal{H}\left(-x_{0}\right) . M_{1}$ is said to be a lower barrier for the pair $(F, f)$, if

$$
F_{\left.\right|_{M_{1}}} \leq f
$$


and $M_{2}$ is called an upper barrier for $(F, f)$, if

$$
F_{\left.\right|_{M_{2}}} \geq f
$$

where in both cases the right-hand side $f$ may either depend on $x \in M_{i}$ or $\nu \in$ $T_{x}\left(S^{n+1}\right)$ for $x \in M_{i}$, or, in the latter case, equivalently on $\tilde{x} \in M_{i}^{*}$.

0.4. Theorem. Let $F \in C^{5}\left(\Gamma_{+}\right)$be a symmetric, positively homogeneous and monotone curvature function such that its inverse $\tilde{F}$ is of class $(K)$, let $0<$ $f \in C^{5}\left(S^{n+1}\right)$, and assume that there exist upper and lower barriers for $(F, f)$ in the hemisphere $\mathcal{H}\left(-x_{0}\right)$ as defined in the Assumption 0.3, where in addition the barriers $M_{i}$ should bound a connected open set $\Omega$ such that the mean curvature vector of $M_{1}$ should point to the exterior of $\Omega$ and the mean curvature vector of $M_{2}$ should point into $\Omega$. Then the dual problems

$$
F_{\left.\right|_{M}}=f(\tilde{x})
$$

and

$$
\tilde{F}_{\left.\right|_{M^{*}}}=f^{-1}(\tilde{x})
$$

have strictly convex solutions $M$ resp. $M^{*}$ of class $C^{6, \alpha}, 0<\alpha<1$, such that the convex bodies $\hat{M}, \hat{M}^{*}$ are strictly contained in the open hemispheres $\mathcal{H}\left(-x_{0}\right)$ resp. $\mathcal{H}\left(x_{0}\right)$.

The paper is organized as follows: Section 1 gives an overview of the definitions and conventions we rely on, while the dual relationship between $M$ and $M^{*}$ and the properties of the Gauß map are derived in Section 2. The curvature estimates are proved in Section 3, the lower order estimates in Section 4. The next two sections contain a uniqueness result for invariant convex hypersurfaces with constant $F$, and the existence proof in the invariance case, which is based on a continuity method using Smale's infinite dimensional version of Sard's theorem [18]. Finally, in Section 7 we prove Theorem 0.4.

0.5. Remark. Let us emphasize that after Section 2 we shall only consider equation (0.11). In order to simplify notation we then shall drop the tilde and the other embellishments and shall solve the equation

$$
F_{\left.\right|_{M}}=f(x)
$$

for a curvature function $F$ of class $(K)$, where we note that we also replaced $f^{-1}$ by $f$.

\section{Notations AND DEFinitions}

The main objective of this section is to state the equations of Gauß, Codazzi, and Weingarten for hypersurfaces $M$ in a $(\mathrm{n}+1)$-dimensional Riemannian manifold $N$. Geometric quantities in $N$ will be denoted by $\left(\bar{g}_{\alpha \beta}\right),\left(\bar{R}_{\alpha \beta \gamma \delta}\right)$, etc., and 
those in $M$ by $\left(g_{i j}\right),\left(R_{i j k l}\right)$, etc. Greek indices range from 0 to $n$ and Latin from 1 to $n$; the summation convention is always used. Generic coordinate systems in $N$ resp. $M$ will be denoted by $\left(x^{\alpha}\right)$ resp. $\left(\xi^{i}\right)$. Covariant differentiation will simply be indicated by indices, only in case of possible ambiguity they will be preceded by a semicolon, i.e., for a function $u$ in $N,\left(u_{\alpha}\right)$ will be the gradient and $\left(u_{\alpha \beta}\right)$ the Hessian, but e.g., the covariant derivative of the curvature tensor will be abbreviated by $\bar{R}_{\alpha \beta \gamma \delta ; \epsilon}$. We also point out that

$$
\bar{R}_{\alpha \beta \gamma \delta ; i}=\bar{R}_{\alpha \beta \gamma \delta ; \epsilon} x_{i}^{\epsilon}
$$

with obvious generalizations to other quantities.

Let $M$ be a $C^{2}$-hypersurface with normal $\nu$.

In local coordinates, $\left(x^{\alpha}\right)$ and $\left(\xi^{i}\right)$, the geometric quantities of the hypersurface $M$ are connected through the following equations

$$
x_{i j}^{\alpha}=-h_{i j} \nu^{\alpha}
$$

the so-called Gauß formula. Here, and also in the sequel, a covariant derivative is always a full tensor, i.e.,

$$
x_{i j}^{\alpha}=x_{, i j}^{\alpha}-\Gamma_{i j}^{k} x_{k}^{\alpha}+\bar{\Gamma}_{\beta \gamma}^{\alpha} x_{i}^{\beta} x_{j}^{\gamma} .
$$

The comma indicates ordinary partial derivatives.

In this implicit definition the second fundamental form $\left(h_{i j}\right)$ is taken with respect to $-\nu$.

The second equation is the Weingarten equation

$$
\nu_{i}^{\alpha}=h_{i}^{k} x_{k}^{\alpha},
$$

where we remember that $\nu_{i}^{\alpha}$ is a full tensor.

Finally, we have the Codazzi equation

$$
h_{i j ; k}-h_{i k ; j}=\bar{R}_{\alpha \beta \gamma \delta} \nu^{\alpha} x_{i}^{\beta} x_{j}^{\gamma} x_{k}^{\delta}
$$

and the Gauß equation

$$
R_{i j k l}=\left\{h_{i k} h_{j l}-h_{i l} h_{j k}\right\}+\bar{R}_{\alpha \beta \gamma \delta} x_{i}^{\alpha} x_{j}^{\beta} x_{k}^{\gamma} x_{l}^{\delta} .
$$

When we consider hypersurfaces $M \subset S^{n+1}$ to be embedded in $\mathbb{R}^{n+2}$, we label the coordinates in $\mathbb{R}^{n+2}$ as $\left(x^{a}\right)$, i.e., indices $a, b, c, \ldots$ always run through $n+2$ values either from 1 to $n+2$ or from 0 to $n+1$.

Let us also state the definition of curvature functions of class $(K)$

1.1. Definition. A symmetric curvature function $F \in C^{2, \alpha}\left(\Gamma_{+}\right) \cap C^{0}\left(\bar{\Gamma}_{+}\right)$positively homogeneous of degree $d_{0}>0$ is said to be of class $(K)$ if

$$
F_{i}=\frac{\partial F}{\partial \kappa^{i}}>0 \text { in } \Gamma_{+},
$$




$$
F_{\left.\right|_{\partial \Gamma_{+}}}=0
$$

and

$$
F^{i j, k l} \eta_{i j} \eta_{k l} \leq F^{-1}\left(F^{i j} \eta_{i j}\right)^{2}-F^{i k} \tilde{h}^{j l} \eta_{i j} \eta_{k l} \quad \forall \eta \in \mathcal{S},
$$

or, equivalently, if we set $\hat{F}=\log F$,

$$
\hat{F}^{i j, k l} \eta_{i j} \eta_{k l} \leq-\hat{F}^{i k} \tilde{h}^{j l} \eta_{i j} \eta_{k l} \quad \forall \eta \in \mathcal{S},
$$

where $F$ is evaluated at $\left(h_{i j}\right)$.

A detailed analysis of these curvature functions can be found in [8, Section 1]. In this paper we actually do not need the full strength of inequality (1.10).

As we have shown in [7, Lemma 1.3 and Remark 1.4] a symmetric curvature function $F \in C^{2}\left(\Gamma_{+}\right)$satisfies inequality (1.9) iff

$$
F_{i} \kappa_{i} \leq F_{j} \kappa_{j}, \quad \text { for } \quad \kappa_{j} \leq \kappa_{i},
$$

and

$$
F_{i j} \xi^{i} \xi^{j} \leq F^{-1}\left(F_{i} \xi^{i}\right)^{2}-F_{i} \kappa_{i}^{-1}\left|\xi^{i}\right|^{2} \quad \forall \xi \in \mathbb{R}^{n},
$$

where $F_{i}, F_{i j}$ are ordinary partial derivatives of $F$ in $\Gamma_{+}$.

We only need the property (1.11).

Let us finish this section with a simple yet useful observation.

1.2. Lemma. Let $F \in(K)$ be homogeneous of degree 1 , then $F$ is concave.

Proof. It suffices to prove that the right-hand side of the inequality (1.12) is non-positive, if $F$ is homogeneous of degree 1.

Using Schwarz's inequality we deduce

$$
\begin{aligned}
F_{i} \xi^{i} & =\sum_{i} F_{i}^{\frac{1}{2}} \kappa_{i}^{\frac{1}{2}} F_{i}^{\frac{1}{2}} \kappa_{i}^{-\frac{1}{2}} \xi^{i} \\
& \leq\left(\sum_{i} F_{i} \kappa_{i}\right)^{\frac{1}{2}}\left(\sum_{i} F_{i} \kappa_{i}^{-1}\left|\xi^{i}\right|^{2}\right)^{\frac{1}{2}}=F^{\frac{1}{2}}\left(\sum_{i} F_{i} \kappa_{i}^{-1}\left|\xi^{i}\right|^{2}\right)^{\frac{1}{2}},
\end{aligned}
$$

hence the result.

\section{Polar Sets}

Let $M \subset S^{n+1}$ be a connected, closed, immersed, strictly convex hypersurface given by an immersion

$$
x: M_{0} \rightarrow M \subset S^{n+1},
$$

then $M$ is embedded, homeomorphic to $S^{n}$, contained in an open hemisphere and is the boundary of a convex body $\hat{M} \subset S^{n+1}$, cf. [3]. 
Considering $M$ as a codimension 2 submanifold of $\mathbb{R}^{n+2}$ such that

$$
x_{i j}=-g_{i j} x-h_{i j} \tilde{x},
$$

where $\tilde{x} \in T_{x}\left(\mathbb{R}^{n+2}\right)$ represents the exterior normal vector $\nu \in T_{x}\left(S^{n+1}\right)$, we want to prove that the mapping

$$
\tilde{x}: M_{0} \rightarrow S^{n+1}
$$

is an embedding of a strictly convex, closed, connected hypersurface $\tilde{M}$. We call this mapping the Gauß map of $M$.

First, we shall show that the Gauß map is injective. To prove this result we need the following lemma.

2.1. Lemma. Let $M \subset S^{n+1}$ be a closed, connected, strictly convex hypersurface and denote by $\hat{M}$ its (closed) convex body. Let $x \in M$ be fixed and $\tilde{x}$ be the corresponding outward normal vector, then

$$
\langle y, \tilde{x}\rangle \leq 0 \quad \forall y \in \hat{M}
$$

and also strictly less than 0 unless $y=x$.

The preceding inequality also characterizes the points in $\hat{M}$, namely, let $y \in$ $S^{n+1}$ be such that

$$
\langle y, \tilde{x}\rangle \leq 0 \quad \forall x \in M
$$

then $y \in \hat{M}$.

Proof. „(2.4)“ First, we note that $\hat{M}$ is contained in an open hemisphere $\mathcal{H}\left(x_{0}\right)$.

Let $y \in \operatorname{int} \hat{M}$ be arbitrary and let $z=z(t), 0 \leq t \leq d$, be the unique minimizing geodesic in $S^{n+1}$ connecting $y$ and $x$ such that

$$
z(0)=x \quad \wedge \quad z(d)=y
$$

parametrized by arc length, and hence $0<d<\pi$.

Viewing $z$ as a curve in $\mathbb{R}^{n+2}$ the geodesic equation has the form

$$
\ddot{z} \equiv \frac{D}{d t} \dot{z}=-z .
$$

If the coordinate system in $\mathbb{R}^{n+2}$ is Euclidean, the covariant derivatives are just ordinary derivatives.

It is well-known that the geodesic $z$ is contained in $\hat{M}$ and that

$$
\langle\dot{z}(0), \tilde{x}\rangle<0 ;
$$

notice that, after introducing geodesic polar coordinates in $S^{n+1}$ centered in $y$, we have

$$
\langle\dot{z}(0), \tilde{x}\rangle=-\left\langle\frac{\partial}{\partial r}, \nu\right\rangle
$$


and hence is strictly negative, cf. the remarks in [5, Section 4] after Theorem 4.6.

Thus, $\varphi(t)=\langle z(t), \tilde{x}\rangle$ satisfies the initial value problem

$$
\ddot{\varphi}=-\varphi, \quad \varphi(0)=0, \quad \dot{\varphi}(0)<0,
$$

and is therefore equal to

$$
\varphi(t)=-\lambda \sin t, \quad \lambda>0
$$

i.e.,

$$
\varphi(t)<0 \quad \forall 0<t<\pi .
$$

Now, let $y \in M, y \neq x$, be arbitrary, and consider a sequence $z_{k}$ of geodesics parametrized in the interval $0 \leq t \leq 1$, such that

$$
z_{k}(0)=x \quad \wedge \quad z_{k}(1) \rightarrow y
$$

where $z_{k}(1) \in \operatorname{int} \hat{M}$.

The geodesics $z_{k}$ converge to a geodesic $z$ connecting $x$ and $y$. If

$$
\langle\dot{z}(0), \tilde{x}\rangle<0,
$$

then the previous arguments are valid yielding

$$
\langle y, \tilde{x}\rangle<0 \text {. }
$$

On the other hand, the alternative

$$
\langle y, \tilde{x}\rangle=0
$$

leads to a contradiction, since then the geodesic $z$ would be part of the tangent space $T_{x}(M)$ which is impossible, cf. the considerations in [5] after the equation (4.17).

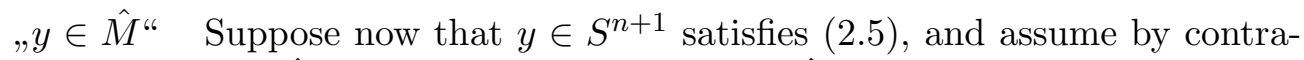
diction that $y \in \mathcal{C} \hat{M}$. Pick an arbitrary $\bar{x}_{0} \in \operatorname{int} \hat{M}, \bar{x}_{0} \neq-y$, and let $z=z(t)$, $0 \leq t \leq d$, be the minimizing geodesic joining $\bar{x}_{0}$ and $y$ parameterized by arc length, such that $z(0)=\bar{x}_{0}$ and $z(d)=y$. The geodesic intersects $M$ in a unique point $x, x=z\left(t_{1}\right), 0<t_{1}<d$.

Define

$$
\varphi(t)=\langle z(t), \tilde{x}\rangle
$$

then

$$
\varphi\left(t_{1}\right)=0 \wedge \dot{\varphi}\left(t_{1}\right)>0
$$

and hence

$$
\varphi(t)=\lambda \sin \left(t-t_{1}\right), \quad \lambda>0,
$$


and we conclude

$$
\varphi(t)>0 \quad \forall t_{1}<t<t_{1}+\pi
$$

contradicting the assumption $\varphi(d) \leq 0$.

Therefore we have proved $y \in \hat{M}$.

2.2. Theorem. Let $x: M_{0} \rightarrow M \subset S^{n+1}$ be the embedding of a closed, connected, strictly convex hypersurface, then the Gauß map defined in (2.3) is injective, where we identify $\mathbb{R}^{n+2}$ with its individual tangent spaces.

Proof. We again assume $M$ to be a codimension 2 submanifold in $\mathbb{R}^{n+2}$. Suppose there would be two points $p_{1} \neq p_{2}$ in $M_{0}$ such that

$$
\tilde{x}\left(p_{1}\right)=\tilde{x}\left(p_{2}\right),
$$

then the function

$$
\varphi(y)=\left\langle y, \tilde{x}\left(p_{1}\right)\right\rangle
$$

would vanish in the points $x\left(p_{1}\right)$ as well as $x\left(p_{2}\right)$ contrary to the results of Lemma 2.1.

2.3. Lemma. As a submanifold of codimension $2 M$ satisfies the Weingarten equations

$$
\tilde{x}_{i}=h_{i}^{k} x_{k}
$$

for the normal $\tilde{x}$ and also

$$
x_{i}=g_{i}^{k} x_{k}
$$

for the normal $x$.

Proof. We only have to prove the non-trivial Weingarten equation.

First we infer from

$$
\langle x, \tilde{x}\rangle=0
$$

that

$$
0=\left\langle x_{i}, \tilde{x}\right\rangle+\left\langle x, \tilde{x}_{i}\right\rangle=\left\langle x, \tilde{x}_{i}\right\rangle .
$$

Furthermore, there holds

$$
0=\left\langle\tilde{x}, \tilde{x}_{i}\right\rangle,
$$

since $\langle\tilde{x}, \tilde{x}\rangle=1$. Hence, we deduce

$$
\tilde{x}_{i}=a_{i}^{k} x_{k} .
$$

Differentiating the relation $\left\langle x_{j}, \tilde{x}\right\rangle=0$ covariantly we obtain

$$
\left\langle\tilde{x}_{j}, x_{i}\right\rangle=h_{i j}
$$


and we infer (2.23) in view of (2.28).

We can now prove

2.4. Theorem. Let $x: M_{0} \rightarrow M \subset S^{n+1}$ be a closed, connected, strictly convex hypersurface of class $C^{m}, m \geq 3$, then the Gauß map $\tilde{x}$ in (2.3) is the embedding of a closed, connected, strictly convex hypersurface $\tilde{M} \subset S^{n+1}$ of class $C^{m-1}$.

Viewing $\tilde{M}$ as a codimension 2 submanifold in $\mathbb{R}^{n+2}$, its Gaussian formula is

$$
\tilde{x}_{i j}=-\tilde{g}_{i j} \tilde{x}-\tilde{h}_{i j} x
$$

where $\tilde{g}_{i j}, \tilde{h}_{i j}$ are the metric and second fundamental form of the hypersurface $\tilde{M} \subset S^{n+1}$, and $x=x(\xi)$ is the embedding of $M$ which also represents the exterior normal vector of $\tilde{M}$. The second fundamental form $\tilde{h}_{i j}$ is defined with respect to the interior normal vector.

The second fundamental forms of $M, \tilde{M}$ and the corresponding principal curvatures $\kappa_{i}, \tilde{\kappa}_{i}$ satisfy

$$
h_{i j}=\tilde{h}_{i j}=\left\langle\tilde{x}_{i}, x_{j}\right\rangle
$$

and

$$
\tilde{\kappa}_{i}=\kappa_{i}^{-1}
$$

Proof. (i) From the Weingarten equation (2.23) we infer

$$
\tilde{g}_{i j}=\left\langle\tilde{x}_{i}, \tilde{x}_{j}\right\rangle=h_{i}^{k} h_{k j}
$$

is positive definite, hence $\tilde{x}=\tilde{x}(\xi)$ is an embedding of a closed, connected hypersurface, where we also used Theorem 2.2 .

(ii) The pair $(x, \tilde{x})$ satisfies

$$
\langle x, \tilde{x}\rangle=0
$$

and we claim that $x$ is the exterior normal vector of $\tilde{M}$ in $\tilde{x}$, where as usual we identify the normal vector $\tilde{\nu}=\left(\tilde{\nu}^{\alpha}\right) \in T_{\tilde{x}}\left(S^{n+1}\right)$ with its embedding in $T_{\tilde{x}}\left(\mathbb{R}^{n+2}\right)$.

Differentiating (2.34) covariantly and using the fact that $\tilde{x}$ is a normal vector for $M$ we deduce

$$
0=\left\langle x, \tilde{x}_{i}\right\rangle
$$

i.e., $\tilde{x}$ and $x$ span the normal space of the codimension 2 submanifold $\tilde{M}$.

Let us define the second fundamental form $\tilde{h}_{i j}$ of $\tilde{M} \subset S^{n+1}$ with respect to the normal vector $\tilde{\nu} \in T_{\tilde{x}}\left(S^{n+1}\right)$ corresponding to $x$, then the codimension 2 Gaussian formula is exactly (2.30). 
Differentiating the Weingarten equation (2.23) covariantly with respect to the metric $\tilde{g}_{i j}$ and indicating the covariant derivatives with respect to $\tilde{g}_{i j}$ by a semicolon and those with respect to $g_{i j}$ simply by indices, we obtain

$$
\tilde{x}_{; i j}=h_{i ; j}^{k} x_{k}+h_{i}^{k} x_{; k j}
$$

and we deduce further

$$
\tilde{h}_{i j}=-\left\langle\tilde{x}_{; i j}, x\right\rangle=-h_{i}^{k}\left\langle x_{k j}, x\right\rangle=h_{i}^{k} g_{k j}=h_{i j} .
$$

On the other hand, we infer from (2.35)

$$
\tilde{h}_{i j}=-\left\langle\tilde{x}_{; i j}, x\right\rangle=\left\langle\tilde{x}_{i}, x_{j}\right\rangle
$$

which proves (2.31).

The last relation (2.32) follows from (2.37) and (2.33).

Finally, the normal vector $x$ must correspond to the exterior normal of $\tilde{M}$ in $T_{\tilde{x}}\left(S^{n+1}\right)$, since $\tilde{h}_{i j}$ is positive definite.

We can also define a Gauß map from the strictly convex, connected, closed hypersurfaces $\tilde{M} \subset S^{n+1}$ into $S^{n+1}$, and the preceding theorem shows that the two Gauß maps are inverse to each other, i.e., if we start with a closed, strictly convex hypersurface $M \subset S^{n+1}$, apply the Gauß map to obtain a strictly convex hypersurface $\tilde{M} \subset S^{n+1}$, and then apply the second Gauß map, then we return to $M$ with a pointwise equality.

Denoting the two Gauß maps simply by a tilde, this can be expressed in the form

$$
x=\tilde{\tilde{x}},
$$

or, equivalently, in the form of a commutative diagram

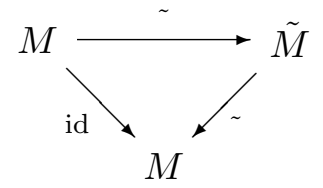

Before we give an equivalent characterization of the images of the Gauß maps, let us show that the images of strictly convex hypersurfaces by the Gauß maps are as smooth as the original hypersurfaces.

2.5. Lemma. Let $M \subset S^{n+1}$ be a closed, connected, strictly convex hypersurface of class $C^{m, \alpha}, m \geq 3,0 \leq \alpha \leq 1$ and let $\tilde{M} \subset S^{n+1}$ be its image under the Gauß map. Let $\tilde{M} \subset \mathcal{H}\left(x_{0}\right)$ and express $\tilde{M}$ as a graph in geodesic polar coordinates $\left(\rho, x^{i}\right)$ centered in $x_{0}, \tilde{M}=$ graph $\tilde{u}_{S^{n}}$, then $\tilde{h}_{i j}$, expressed in corresponding local coordinates $x^{i}$ of $S^{n}$, is of class $C^{m-2, \alpha}$. 
Proof. Notice that this is a non-trivial statement, since $\tilde{M}$ is only known to be of class $C^{m-1, \alpha}$.

Let $\left(x^{a}\right)=\left(x^{0}, x^{i}\right)$ be Euclidean coordinates in $\mathbb{R}^{n+2}$ and assume without loss of generality that $x_{0}=(1,0)$. Writing $x=\left(x^{0}, z\right), z \in \mathbb{R}^{n+1}$, we have

$$
\left|x^{0}\right|^{2}=1-|z|^{2} \quad \forall x \in S^{n+1},
$$

i.e., after introducing Euclidean polar coordinates $\left(r, x^{i}\right)$ in $\mathbb{R}^{n+1}$, the hemisphere $\mathcal{H}\left(x_{0}\right)$ is given as the embedding

$$
x=\left(x^{0}, r, x^{i}\right)=\left(\sqrt{1-r^{2}}, r, x^{i}\right)
$$

and the lower hemisphere $\mathcal{H}\left(-x_{0}\right)$ by the embedding

$$
x=\left(x^{0}, r, x^{i}\right)=\left(-\sqrt{1-r^{2}}, r, x^{i}\right) .
$$

The metric in $S^{n+1} \backslash\left\{x^{0}=0\right\}$ is then expressed as

$$
d \bar{s}^{2}=\frac{1}{1-r^{2}} d r^{2}+r^{2} \sigma_{i j} d x^{i} d x^{j}
$$

where $\sigma_{i j}$ is the metric of $S^{n}$.

Defining $\rho$ by

$$
d \rho=\frac{1}{\sqrt{1-r^{2}}} d r \quad \wedge \quad \rho(0)=0
$$

will give us geodesic polar coordinates $\left(\rho, x^{i}\right)$ in $\mathcal{H}\left(x_{0}\right)$ centered in $x_{0}$.

Now, assuming $\tilde{M} \subset \mathcal{H}\left(x_{0}\right)$ implies $M \subset \mathcal{H}\left(-x_{0}\right)$, in view of Lemma 2.1. Let $\left(\xi^{i}\right)$ be local coordinates for $M$ and express the Gauß map $\tilde{x}(\xi)$ in the coordinates in $(2.42)$

$$
\tilde{x}(\xi)=\left(x^{0}(\xi), r(\xi), x^{i}(\xi)\right),
$$

then

$$
r(\xi)=u\left(x^{i}(\xi)\right) \wedge x^{0}(\xi)=\sqrt{1-u^{2}\left(x^{i}(\xi)\right)},
$$

where $\tilde{M}$ has been written as a graph over $S^{n}$

$$
\tilde{M}=\left\{r=u\left(x^{i}\right):\left(x^{i}\right) \in S^{n}\right\} ;
$$

notice that in geodesic polar coordinates we have $\tilde{M}=\operatorname{graph} \tilde{u}$ with

$$
\tilde{u}=\rho(u) .
$$

In the coordinates $\left(\xi^{i}\right)$ the second fundamental form $\tilde{h}_{i j}$ is already known to be of class $C^{m-2, \alpha}$ because of the relation (2.31). Hence the lemma will be proved, if we can show that the transformation $\left(x^{i}(\xi)\right)$ is a $C^{m-1, \alpha}$-diffeomorphism, i.e., we have to show that the Jacobian is invertible. 
Now, the induced metric $\tilde{g}_{i j}$ can be expressed as

$$
\begin{aligned}
\tilde{g}_{i j} & =\left\langle\tilde{x}_{i}, \tilde{x}_{j}\right\rangle=x_{i}^{0} x_{j}^{0}+r_{i} r_{j}+r^{2} \sigma_{k l} x_{i}^{k} x_{j}^{k} \\
& =\frac{1}{1-r^{2}} r_{i} r_{j}+r^{2} \sigma_{k l} x_{i}^{k} x_{j}^{l} \\
& =\left\{\frac{1}{1-u^{2}} u_{k} u_{l}+u^{2} \sigma_{k l}\right\} x_{i}^{k} x_{j}^{l},
\end{aligned}
$$

hence $\left(x_{i}^{k}\right)$ is invertible, since the left-hand side of this equation has this property.

2.6. Theorem. Let $M \subset S^{n+1}$ be a closed, connected, strictly convex hypersurface of class $C^{m, \alpha}, m \geq 2,0 \leq \alpha \leq 1$, then $\tilde{M} \subset N$, its image under the Gauß map is also of class $C^{m, \alpha}$.

Proof. (i) First, let us assume that $m \geq 3$ and $0 \leq \alpha \leq 1$. The Gauß map is then of class $C^{m-1, \alpha}$, i.e., $\tilde{M}$ is of class $C^{m-1, \alpha}$. Here, we use the coordinates $\left(\xi^{i}\right)$ for $M$ also as coordinates for $\tilde{M}$. The metric $\tilde{g}_{i j}$ and the Christoffel symbols of $\tilde{M}$ are then of class $C^{m-2, \alpha}$ resp. $C^{m-3, \alpha}$, while the second fundamental form $\tilde{h}_{i j}$ is of class $C^{m-2, \alpha}$, in view of $(2.31)$.

We may assume that $M \subset \mathcal{H}\left(-x_{0}\right)$ and $\tilde{M} \subset \mathcal{H}\left(x_{0}\right)$, where $x_{0}=(1,0)$. Using then geodesic polar coordinates $\left(\rho, \xi^{i}\right)$ centered in $x_{0}$, the metric in $S^{n+1}$ can be expressed in the form

$$
d \bar{s}^{2}=d \rho^{2}+e^{2 \psi(\rho)} \sigma_{i j} d \xi^{i} d \xi^{j}
$$

or, in conformal coordinates

$$
d \bar{s}^{2}=e^{2 \psi(\rho)}\left\{d \tau^{2}+\sigma_{i j} d \xi^{i} d \xi^{j}\right\} .
$$

Writing $\tilde{M}$ as a graph in the coordinates $\left(\tau, \xi^{i}\right)$

$$
\tilde{M}=\operatorname{graph} u_{\left.\right|^{n}},
$$

the second fundamental form $h_{i j}$ of $\tilde{M}$ can be expressed as

$$
e^{-\psi} v^{-1} h_{i j}=-u_{i j}-\bar{\Gamma}_{00}^{0} u_{i} u_{j}-\bar{\Gamma}_{0 i}^{0} u_{j}-\bar{\Gamma}_{0 j}^{0} u_{i}-\bar{\Gamma}_{i j}^{0},
$$

where

$$
v^{2}=1+\sigma^{i j} u_{i} u_{j}
$$

and where we note that the second fundamental form $h_{i j}$ is of class $C^{m-2, \alpha}$, cf. Lemma 2.5.

We want to replace the covariant derivatives $u_{i j}$ of $u$ with the covariant derivatives $u_{; i j}$ of $u$ with respect to the metric $\sigma_{i j}$ to deduce that $u_{; i j}$ is of class $C^{m-2, \alpha}$, and hence $u \in C^{m, \alpha}\left(S^{n}\right)$.

To achieve this we define a new metric $\hat{g}_{\alpha \beta}$ in the ambient space

$$
\hat{g}_{\alpha \beta}=e^{-2 \psi} \bar{g}_{\alpha \beta} \text {, }
$$


where $\bar{g}_{\alpha \beta}$ is the metric in (2.52). Let $\hat{g}_{i j}, \hat{h}_{i j}$ and $\hat{\nu}$ be the obvious geometric quantities of $\tilde{M}$ with respect to the new metric, then there holds

$$
h_{i j} e^{-\psi}=\hat{h}_{i j}+\psi_{\alpha} \hat{\nu}^{\alpha} \hat{g}_{i j}
$$

as one easily checks.

On the other hand, $\hat{h}_{i j}$ can be expressed in terms of the Hessian $u_{; i j}$ of $u$ with respect to the metric $\sigma_{i j}$, namely,

$$
\hat{h}_{i j}=-u_{; i j} v^{-1}
$$

$$
h_{i j} e^{-\psi}=-u_{; i j} v^{-1}+\psi_{\alpha} \hat{\nu}^{\alpha}\left(u_{i} u_{j}+\sigma_{i j}\right),
$$

hence, $u_{; i j}$ is of class $C^{m-2, \alpha}$.

(ii) The case $m=2$ and $0 \leq \alpha \leq 1$ follows by approximation and the uniform $C^{2, \alpha}$-estimates. Notice that the approximating second fundamental forms will converge in $C^{0}$.

2.7. Definition. (i) Let $M \subset S^{n+1}$ be a closed, connected, strictly convex hypersurface, then we define its polar set $M^{*} \subset S^{n+1}$ by

$$
M^{*}=\left\{y \in S^{n+1}: \sup _{x \in M}\langle x, y\rangle=0\right\}
$$

where the scalar product is the scalar product in $\mathbb{R}^{n+2}$ and $x, y$ are Euclidean coordinates.

(ii) Let $\hat{M}$ be the convex body of $M \subset S^{n+1}$, then we define the polar of $\hat{M}$ by

$$
\hat{M}^{*}=\left\{y \in S^{n+1}: \sup _{x \in \hat{M}}\langle x, y\rangle \leq 0\right\} .
$$

2.8. Theorem. The $M \subset S^{n+1}$ be a closed, connected and strictly convex hypersurface, then

$$
M^{*}=\tilde{M}
$$

and

$$
\hat{M}^{*}=\hat{\tilde{M}}
$$

Proof. „(2.62)“ In view of Lemma 2.1 there holds

$$
\tilde{M} \subset M^{*} \text {. }
$$

On the other hand, let $y \in M^{*}$ and $x \in M$ be such that

$$
\langle x, y\rangle=0 .
$$


Then we deduce, after introducing local coordinates in $M$,

$$
\left\langle x_{i}, y\right\rangle=0
$$

and

$$
\left\langle x_{i j}, y\right\rangle \leq 0,
$$

where the derivatives are covariant derivatives with respect to the induced metric $g_{i j}$ of $M$ being viewed as a codimension 2 submanifold.

Combining (2.65) and (2.66) we infer

$$
y= \pm \tilde{x},
$$

but because of $(2.2)$ and (2.67) we deduce $y=\tilde{x}$.

„(2.63)“ In view of Lemma 2.1 we immediately deduce

$$
\hat{M}^{*} \subset \hat{\tilde{M}}
$$

hence we only have to prove the reverse inclusion.

Let $y \in \hat{M}$ and $\tilde{x}, \tilde{z} \in \tilde{M}, \tilde{x} \neq \tilde{z}$, be arbitrary and let $z=z(t)$ be the minimizing geodesic connecting $z(0)=\tilde{x}$ and $z(d)=\tilde{z}$ parametrized by arc length. Then it suffices to prove

$$
\varphi(t)=\langle y, z(t)\rangle \leq 0 \quad \forall 0 \leq t \leq d<\pi .
$$

Assume by contradiction that there exists $0<t_{0}<d$ such that

$$
0<\varphi\left(t_{0}\right)=\sup \{\varphi(t): 0 \leq t \leq d\},
$$

then $\varphi$ solves the initial value problem

$$
\ddot{\varphi}=-\varphi, \quad \varphi\left(t_{0}\right)>0, \quad \dot{\varphi}\left(t_{0}\right)=0,
$$

and hence, it is equal to

$$
\varphi(t)=\lambda \cos \left(t-t_{0}\right) \quad \lambda>0,
$$

contradicting the relations $\varphi(0) \leq 0$ and $\varphi(d) \leq 0$, cf. Lemma 2.1, since there holds

$$
0<t_{0}<\frac{\pi}{2} \quad \vee \quad 0<d-t_{0}<\frac{\pi}{2} .
$$

An important corollary is

2.9. Corollary. (i) Let $M_{i}, i=1,2$, be connected, closed, strictly convex hypersurfaces in $S^{n+1}$, then

$$
\hat{M}_{1} \subset \hat{M}_{2} \Longrightarrow \hat{M}_{2}^{*} \subset \hat{M}_{1}^{*}
$$


(ii) Let $B_{r}\left(x_{0}\right) \subset \mathcal{H}\left(x_{0}\right)$ be a geodesic ball of radius $0<r<\frac{\pi}{2}$, then its polar set is a closed geodesic ball centered in $-x_{0}$

$$
B_{r}\left(x_{0}\right)^{*}=\bar{B}_{r^{*}}\left(-x_{0}\right), \quad 0<r^{*}=\varphi(r)<\frac{\pi}{2},
$$

where $\varphi$ is continuous function.

Proof. We only need to prove (2.76). But since the convex body of a geodesic sphere is the corresponding closed geodesic ball, it suffices to prove that the polar of a geodesic sphere $S_{r}\left(x_{0}\right)$ is a geodesic sphere $S_{r^{*}}\left(-x_{0}\right)$.

Let $\tilde{M}$ be the polar of $S_{r}\left(x_{0}\right)$, then we deduce from (2.31), that $\tilde{M}$ is totally umbilic and hence a geodesic sphere, cf. Section 5 for details. This sphere must be centered in $-x_{0}$, since it is invariant under all $A \in O(n+2)$ having $x_{0}$ as a fixed point.

To conclude this section, we note that, with the help of the Gauß map, the Minkowski type equation

$$
F_{\left.\right|_{M}}=f(\nu)
$$

in $S^{n+1}$ can be expressed in the form

$$
F_{\left.\right|_{M}}=f(\tilde{x}),
$$

where $f$ is supposed to be defined in $S^{n+1}$, or more precisely, in $T_{x}\left(\mathbb{R}^{n+2}\right) \equiv \mathbb{R}^{n+2}$, the latter can be achieved by extending $f$ homogeneously of degree 0 .

Let $M^{*}$ be the polar set of $M, \tilde{F}$ the inverse of $F$, then the equation (2.78) is equivalent to

$$
\tilde{F}_{\left.\right|_{M^{*}}}=f^{-1}(\tilde{x}),
$$

where this time the right-hand side is looked at to be a function defined in the ambient space of $M^{*}$. Solving one equation is equivalent to solving the other.

\section{Curvature estimates}

We prove curvature estimates for the polar hypersurface $M^{*}$ satisfying the equation (2.79). Since neither the result nor its proof relies on the fact that the underlying hypersurface is a polar hypersurface, we consider in this and in the following sections a strictly convex hypersurface $M$ satisfying the equation

$$
F_{\left.\right|_{M}}=f(x) \quad \forall x \in M,
$$

where $0<f \in C^{5}\left(S^{n+1}\right)$ and $F \in(K)$ of class $C^{5}$, and we shall prove that this problem has a solution, if Assumption 0.1 is satisfied. Since any positive power of $F$ is again of class $(K)$, we shall assume that $F$ is homogeneous of degree 1 and hence concave, cf. Lemma 1.2. 
3.1. Theorem. Let $M \in C^{4, \alpha}$ be a strictly convex hypersurface in $S^{n+1}$ satisfying the equation (3.1), then its principal curvatures $\kappa_{i}$ are uniformly bounded, i.e., there exist positive constants $c_{1}, c_{2}$ such that

$$
0<c_{1} \leq \kappa_{i} \leq c_{2} \quad \forall 1 \leq i \leq n,
$$

where the $c_{i}$ only depend on $F$ and $f$, which are supposed to satisfy the requirements mentioned above.

Proof. It suffices to prove the upper estimate, since the lower estimate follows from the fact that $F$ is continuous in $\bar{\Gamma}_{+}$and vanishes on the boundary.

The second fundamental form $h_{i j}$ satisfies the equation

$$
\begin{aligned}
-F^{k l} h_{j ; k l}^{i}= & F^{k l} h_{k r} h_{l}^{r} h_{j}^{i}-F h^{k i} h_{k j}+F^{k l, r s} h_{k l ; j} h_{r s ; m} g^{m i} \\
& -f_{\alpha \beta} x_{k}^{\alpha} x_{j}^{\beta} g^{k i}+f_{\alpha} \nu^{\alpha} h_{j}^{i}+F \delta_{j}^{i}-F^{k l} g_{k l} h_{j}^{i},
\end{aligned}
$$

cf. the corresponding equation in [6, equ. (5.4)], where an evolution problem is considered. The present situation can be recovered by setting $\dot{\Phi}=1, \ddot{\Phi}=0$, $\tilde{f}=f, \Phi-\tilde{f}=0$ and $K_{N}=1$.

We want to apply the maximum principle to obtain an a priori estimate for

$$
\varphi=\sup \left\{h_{i j} \eta^{i} \eta^{j}:\|\eta\|=1\right\} .
$$

Let $x_{0} \in M$ be a point where $\varphi$ attains its maximum. We then introduce Riemannian normal coordinates $\xi^{i}$ at $x_{0}$ such that at $x_{0}=x\left(\xi_{0}\right)$ we have

$$
g_{i j}=\delta_{i j}, \quad h_{i j}=\kappa_{i} \delta_{i j} \quad \text { and } \quad \varphi=h_{n}^{n} .
$$

Let $\eta=\left(\eta^{i}\right)$ be the contravariant vector field defined by

$$
\eta=(0, \ldots, 1)
$$

in a neighbourhood of $\xi_{0}$ and set

$$
\tilde{\varphi}=\frac{h_{i j} \eta^{i} \eta^{j}}{g_{i j} \eta^{i} \eta^{j}}
$$

$\tilde{\varphi}$ is well defined in a neighbourhood of $\xi_{0}$.

Now $\tilde{\varphi}$ assumes its maximum at $\xi=\xi_{0}$. Moreover, at $\xi=\xi_{0}$ the covariant derivatives up to order two of $\tilde{\varphi}$ coincide with those of $h_{n}^{n}$, i.e., $\tilde{\varphi}$ satisfies the same differential equation at $\xi_{0}$ as $h_{n}^{n}$. For the sake of greater clarity let us therefore treat $h_{n}^{n}$ like a scalar and pretend that $\varphi$ is defined by

$$
\varphi=h_{n}^{n} \text {. }
$$


Applying the maximum principle in $\xi_{0}$ we deduce

$$
\begin{aligned}
0 \leq & F^{k l} h_{k r} h_{l}^{r} h_{n}^{n}-F\left|h_{n}^{n}\right|^{2}+F^{k l, r s} h_{k l ; n} h_{r s ; m} g^{m n} \\
& -f_{\alpha \beta} x_{k}^{\alpha} x_{n}^{\beta} g^{k n}+f_{\alpha} \nu^{\alpha} h_{n}^{n}+F-F^{k l} g_{k l} h_{n}^{n},
\end{aligned}
$$

yielding

$$
0 \leq F^{k l} h_{k r} h_{l}^{r} h_{n}^{n}-F\left|h_{n}^{n}\right|^{2}+c_{0}\left(1+h_{n}^{n}\right)-F^{k l} g_{k l} h_{n}^{n},
$$

where

$$
c_{0}=c_{0}\left(|f|_{2,0}\right) .
$$

The function $F$ is of class $(K)$ and thus satisfies the estimate (1.11). Let $\kappa_{1}$ be the smallest principal curvature of $M$ in $x_{0}$, then

$$
F=\sum_{i} F_{i} \kappa_{i} \leq n F_{1} \kappa_{1}
$$

and hence

$$
\begin{aligned}
F^{k l} h_{k}^{r} h_{r l} h_{n}^{n}-F\left|h_{n}^{n}\right|^{2} & =F_{1} \kappa_{1}\left(\kappa_{1}-\kappa_{n}\right) \kappa_{n}+\sum_{i=2}^{n} F_{i} \kappa_{i}\left(\kappa_{i}-\kappa_{n}\right) \kappa_{n} \\
& \leq-\frac{1}{n} F\left(\kappa_{n}-\kappa_{1}\right) \kappa_{n}
\end{aligned}
$$

Now, if $\kappa_{n}$ is supposed to be large in $x_{0}$, then

$$
\kappa_{1} \leq \frac{\kappa_{n}}{2},
$$

because $F=f$ is bounded, hence $\kappa_{n}=h_{n}^{n}$ is a priori bounded.

\section{LOWER ORDER BOUNDS}

To derive the lower bounds we use the group invariance assumption. Let $M \subset S^{n+1}$ be a strictly convex, closed hypersurface and suppose that $M$ is invariant with respect to the group $G \subset O(n+2)$

$$
A M \subset M \quad \forall A \in G \text {. }
$$

Assume furthermore that a common fixed point $x_{0}$ of $G$ is an interior point of $\hat{M}$. The principal curvatures $\kappa_{i}$ of $M$ are then also invariant with respect to $G$, i.e.,

$$
\kappa_{i}(x)=\kappa_{i}(A x) \quad \forall x \in M, \forall A \in G,
$$

as one easily checks. 
Representing $M$ in geodesic polar coordinates with center $x_{0}$ as a graph $u=$ $u(\xi)$ over $S^{n}$, we conclude that the function $u$ is also invariant with respect to the induced isometry group in $S^{n}$, still denoted by $G$, i.e.,

$$
u(\xi)=u(A \xi) \quad \forall \xi \in S^{n}, \forall A \in G .
$$

Since by assumption the induced group has no fixed points in $S^{n}, u$ is orthogonal to the first eigenfunctions of the Laplace operator in $S^{n}$, i.e.,

$$
\int_{S^{n}} x^{i} u=0 \quad \forall 1 \leq i \leq n+1
$$

cf. [9, Proposition 2.5]. Let us state this result as lemma.

4.1. Lemma. Let $u \in C^{0}\left(S^{n}\right)$ be invariant with respect to the induced group $G$, then $u$ is orthogonal to the spherical harmonics of degree 1.

Now, we use stereographic projection $\pi$ to compare $M$ with a strictly convex hypersurface $\pi(M) \subset \mathbb{R}^{n+1}$. Let $-x_{0}$ be the north pole of $S^{n+1}$ and assume that $\hat{M}$ is contained in the lower open hemisphere $\mathcal{H}\left(x_{0}\right)$

$$
\hat{M} \subset \mathcal{H}\left(x_{0}\right)
$$

such that $x_{0} \in \operatorname{int} \hat{M}$, notice that by definition a convex body is always closed. The metric $\bar{g}_{\alpha \beta}$ of $S^{n+1}$ is then conformal to the Euclidean metric

$$
\bar{g}_{\alpha \beta}=\frac{1}{\left(1+\frac{1}{4}|x|^{2}\right)^{2}} \delta_{\alpha \beta},
$$

where $x=\left(x^{\alpha}\right)$ are Euclidean coordinates in $\mathbb{R}^{n+1}$.

The point $x_{0} \in S^{n+1}$ corresponds to the origin $0 \in \mathbb{R}^{n+1}$ and, introducing Euclidean polar coordinates $\left(\rho, \xi^{i}\right)$, the metric in $S^{n+1}$ is expressed as

$$
d \bar{s}^{2}=\frac{1}{\left(1+\frac{1}{4} \rho^{2}\right)^{2}}\left\{d \rho^{2}+\rho^{2} \sigma_{i j} d \xi^{i} d \xi^{j}\right\} .
$$

Comparing this expression with the representation of $\bar{g}_{\alpha \beta}$ in geodesic polar coordinates $\left(r, \xi^{i}\right)$ centered in $x_{0}$, namely,

$$
d \bar{s}^{2}=d r^{2}+h(r) \sigma_{i j} d \xi^{i} d \xi^{j}
$$

and observing that the radial geodesics in $S^{n+1}$ are mapped onto the radial geodesics in $\mathbb{R}^{n+1}$ we deduce that

$$
r=\int_{0}^{\rho} \frac{1}{1+\frac{1}{4} t^{2}}=2 \arctan \frac{\rho}{2} .
$$

Finally, defining

$$
\tau=\log \rho,
$$


we can express the metric in $S^{n+1}$ as

$$
d \bar{s}^{2}=\frac{\rho^{2}}{\left(1+\frac{1}{4} \rho^{2}\right)^{2}}\left\{d \tau^{2}+\sigma_{i j} d \xi^{i} d \xi^{j}\right\} .
$$

Writing $M$ in these coordinates as a graph over $S^{n}$

$$
M=\operatorname{graph} u_{S^{n}}
$$

$u$ is still invariant with respect to the induced group, and graph $u$ also represents $\pi(M)$.

Let

$$
\psi=-\log \left(1+\frac{1}{4} \rho^{2}\right)
$$

such that

$$
\bar{g}_{\alpha \beta}=e^{2 \psi} \hat{g}_{\alpha \beta},
$$

where $\left(\hat{g}_{\alpha \beta}\right)$ is the Euclidean metric, then the respective second fundamental forms $h_{i j}$ and $\hat{h}_{i j}$ are related by

$$
e^{\psi} h_{i}^{j}=\hat{h}_{i}^{j}+\psi_{\alpha} \nu^{\alpha} \delta_{i}^{j},
$$

where $\nu$ is the exterior normal of $\pi(M)$ and

$$
\psi_{\alpha} \nu^{\alpha}=\psi_{0} \nu^{0}=-\frac{1}{2} \frac{\rho}{1+\frac{1}{4} \rho^{2}} v^{-1},
$$

with

$$
v^{2}=1+\sigma^{i j} u_{i} u_{j}
$$

Thus, $\hat{h}_{i j}$ is also positive definite and invariant with respect to the induced group, as is the metric

$$
\hat{g}_{i j}=e^{2 u}\left\{u_{i} u_{j}+\sigma_{i j}\right\} .
$$

Moreover, since $\hat{M}$ is contained in the lower hemisphere, we have

$$
0 \leq r \leq \frac{\pi}{2}
$$

and hence

$$
\rho \leq 2 \tan \frac{\pi}{4}=2
$$

Thus, if the principal curvatures of $M$ are bounded by

$$
0<k_{1} \leq \kappa_{i} \leq k_{2},
$$

then those of $\pi(M)$ are bounded by

$$
0<\hat{k}_{1} \leq \hat{\kappa}_{i} \leq \hat{k}_{2},
$$


where

$$
\hat{k}_{j}=\hat{k}_{j}\left(k_{1}, k_{2}\right), \quad j=1,2 .
$$

Now we can prove that the convex body of $\pi(M)$ contains a Euclidean ball $B_{\rho_{0}}(0)$ and therefore $\hat{M}$ a geodesic ball $B_{r_{0}}\left(x_{0}\right)$.

4.2. Lemma. Assume $x_{0} \in \operatorname{int} \hat{M}, \hat{M} \subset \mathcal{H}\left(x_{0}\right)$, that $M$ is invariant with respect to the group $G$ and the principal curvatures satisfy the estimate (4.21). Then there exists $0<r_{0}=r_{0}\left(k_{1}, k_{2}\right)$ such that the geodesic ball

$$
B_{r_{0}}\left(x_{0}\right) \Subset \operatorname{int} \hat{M} \text {. }
$$

Proof. We shall prove that there exists a Euclidean ball of radius $0<\rho_{0}=$ $\rho_{0}\left(\hat{k}_{1}, \hat{k}_{2}\right)$ such that

$$
B_{\rho_{0}}(0) \Subset \operatorname{int} \widehat{\pi(M)} .
$$

Let $\hat{K}$ be the Gaussian curvature of $\pi(M)=\operatorname{graph} u$, then $u, \hat{g}_{i j}$ and

$$
\hat{K}=\hat{K}(u, \xi)
$$

are invariant functions in $S^{n}$ with respect to the induced group $G$, and hence orthogonal to the spherical harmonics of degree 1, cf. Lemma 4.1. Hence the Steiner point $p$ of $\pi(M)$ coincides with the origin, since in Euclidean coordinates

$$
\begin{aligned}
p^{i} & =\frac{1}{\left|S^{n}\right|} \int_{\pi(M)} x^{i} \hat{K} \\
& =\frac{1}{\left|S^{n}\right|} \int_{S^{n}} x^{i} e^{u} \hat{K} v=0 .
\end{aligned}
$$

The relation (4.25) is then proved in [2]. A similar, more general, result was later proved in [16].

Let $M^{*}$ be the polar hypersurface of $M$, which is then also invariant with respect to the group $G$, since

$$
0=\langle x, \tilde{x}\rangle=\langle A x, A \tilde{x}\rangle \quad \forall x \in M, \forall A \in G .
$$

Then we shall prove

4.3. Lemma. Let $M \subset \mathcal{H}\left(x_{0}\right)$ be a strictly convex hypersurface satisfying the assumptions of Lemma 4.2. Then the polar convex body $\hat{M}^{*}$ of $\hat{M}$ is contained in $\mathcal{H}\left(-x_{0}\right)$ and there exist radii $0<r_{1}^{*}<r_{0}^{*}<\frac{\pi}{2}$ such that

$$
B_{r_{1}^{*}}\left(-x_{0}\right) \Subset \operatorname{int} \hat{M}^{*} \Subset B_{r_{0}^{*}}\left(-x_{0}\right) \Subset \mathcal{H}\left(-x_{0}\right) .
$$


Proof. Since $\hat{M}$ is compact there exists a geodesic ball $B_{r_{1}}\left(x_{0}\right)$ such that

$$
\hat{M} \subset B_{r_{1}}\left(x_{0}\right) \Subset \mathcal{H}\left(x_{0}\right) .
$$

Moreover, due to Lemma 4.2, there exists a geodesic ball $B_{r_{0}}\left(x_{0}\right)$ such that

$$
B_{r_{0}}\left(x_{0}\right) \Subset \operatorname{int} \hat{M},
$$

hence we conclude

$$
B_{r_{1}^{*}}\left(-x_{0}\right)=\operatorname{int} B_{r_{1}}^{*}\left(x_{0}\right) \Subset \operatorname{int} \hat{M}^{*} \Subset B_{r_{0}}^{*}\left(x_{0}\right)=\bar{B}_{r_{0}^{*}}\left(-x_{0}\right) \Subset \mathcal{H}\left(-x_{0}\right) .
$$

Combining the two lemmata, and having in mind that both $M$ and $M^{*}$ are invariant with respect to $G$, so that Lemma 4.2 can be applied to $M$ as well as $M^{*}$, we obtain

4.4. Theorem. Let $M \subset \mathcal{H}\left(x_{0}\right)$ be a strictly convex hypersurface, invariant with respect to the group $G$ and assume that $x_{0} \in \operatorname{int} \hat{M}$ and that the principal curvatures $\kappa_{i}$ satisfy the estimate (4.21), then there exist radii $0<r_{0}<r_{1}<\frac{\pi}{2}$, depending only on the constants $k_{j}, j=1,2$, in (4.21) such that

$$
B_{r_{0}}\left(x_{0}\right) \Subset \operatorname{int} \hat{M} \Subset B_{r_{1}}\left(x_{0}\right) .
$$

The dual relation then also holds for $\hat{M}^{*}$, namely,

$$
B_{r_{1}^{*}}\left(-x_{0}\right) \Subset \operatorname{int} \hat{M}^{*} \Subset B_{r_{0}^{*}}\left(-x_{0}\right),
$$

where

$$
\bar{B}_{r_{i}^{*}}\left(-x_{0}\right)=B_{r_{i}}^{*}\left(x_{0}\right), \quad i=0,1,
$$

and $0<r_{1}^{*}<r_{0}^{*}<\frac{\pi}{2}$.

\section{A uniqueness Result}

In this section we shall show that a strictly convex solution $M \subset S^{n+1}$ of the equation

$$
F=c \equiv \text { const }>0,
$$

where $F$ is an arbitrary curvature function, homogeneous of degree 1 and concave, is a geodesic sphere; notice that a curvature function is always supposed to be symmetric and monotone.

5.1. Theorem. Let $M \subset S^{n+1}$ be a closed strictly convex solution of (5.1), then $M$ is a geodesic sphere. Assuming that $M$ is invariant with respect to the group $G$ and contained in $\mathcal{H}\left(x_{0}\right)$, where $x_{0}$ is a fixed point of $G$, then $M$ has to be a geodesic sphere with center in $x_{0}$. 
Proof. Assume without loss of generality that

$$
F(1, \ldots, 1)=n
$$

and consider the equation (3.3) for the second fundamental form. At a point $\bar{x} \in M$, where

$$
\sup _{M} \max _{i} \kappa_{i}=\kappa_{n}=h_{n}^{n}
$$

is attained, the maximum principle yields, compare the proof of Theorem 3.1,

$$
\begin{aligned}
0 & \leq F^{k l} h_{k r} h_{l}^{r} h_{n}^{n}-F\left|h_{n}^{n}\right|^{2}+F-F^{k l} g_{k l} h_{n}^{n} \\
& =\sum_{i} F_{i} \kappa_{i}\left(\kappa_{i}-\kappa_{n}\right) \kappa_{n}+\sum_{i} F_{i}\left(\kappa_{i}-\kappa_{n}\right) \leq 0 .
\end{aligned}
$$

Hence $\bar{x}$ must be an umbilic and

$$
c=F(\kappa, \ldots, \kappa)=\kappa n,
$$

$$
\sup _{M} \max _{i} \kappa_{i}=\frac{c}{n}
$$

But then all other points have to be umbilics too, since

$$
c=F\left(\kappa_{i}\right) \leq F\left(\frac{c}{n}, \ldots, \frac{c}{n}\right)=c .
$$

Now, any convex umbilic hypersurface $M$ of $S^{n+1}$ has to be a geodesic sphere, as can be most easily seen by choosing a point $y_{0} \in \hat{M}$ and using stereographic projection as in Section 4. From equation (4.15) we then deduce that the projected hypersurface in Euclidean space is also umbilic and hence a sphere, cf. [19, Vol. IV, p. 11].

If $M$ is invariant with respect to $G$ and contained in $\mathcal{H}\left(x_{0}\right)$, then its polar $M^{*}$ is also a strictly convex umbilic hypersurface such that its convex body contains a geodesic ball centered in $-x_{0}$ in its interior

$$
B_{r_{0}^{*}}\left(-x_{0}\right) \Subset \operatorname{int} \hat{M}^{*},
$$

since $\hat{M}$ is contained in a geodesic ball $B_{r_{1}}\left(x_{0}\right) \Subset \mathcal{H}\left(x_{0}\right)$, in view of the compactness of $\hat{M}$ and the assumption $\hat{M} \subset \mathcal{H}\left(x_{0}\right)$. Now for our purpose $M^{*}$ is as good as $M$, thus let us assume without loss of generality that $B_{r_{0}}\left(x_{0}\right) \Subset \hat{M}$ and let us discard the assumption $\hat{M} \Subset \mathcal{H}\left(x_{0}\right)$, since the corresponding result isn't known yet for $\hat{M}^{*}$.

Looking again at the stereographic projection $\pi(M)$ of $M$, where $x_{0}$ is now the south pole, i.e., $\pi\left(x_{0}\right)=0$, we still deduce that $\pi(M)$ is umbilic and hence a sphere, which now is invariant with respect to the group $G$. But as in the proof 
of Lemma 4.2 we can then show that the Steiner point of $\pi(M)$ is the origin, and hence the origin must be the center of the sphere as one easily checks.

We then conclude that $M$ is a "geodesic" sphere centered in $x_{0}$ by the properties of the stereographic projection. Using now the convexity of $M$ and the fact that $x_{0}$ is supposed to be part of $\hat{M}$, we obtain the final result that $\hat{M} \subset \mathcal{H}\left(x_{0}\right)$ and that $M$ is a geodesic sphere centered in $x_{0}$.

\section{Existence of A SOlution}

The existence is proved by a continuity method using Smale's infinite dimensional version of Sard's theorem [18]. Writing the strictly convex hypersurfaces as graphs over $S^{n}$ it is convenient to express the differential operator

$$
F=F\left(h_{i j}\right)=F_{\left.\right|_{M}}
$$

in terms of the standard Levi-Cività connection in $S^{n}$.

Let $x_{0} \in S^{n}$ be a fixed point for the group $G$ and $\mathcal{H}\left(x_{0}\right)$ the corresponding hemisphere. Introducing geodesic polar coordinates centered in $x_{0}$, the metric in $\mathcal{H}\left(x_{0}\right) \backslash\left\{x_{0}\right\}$ can be expressed as

$$
d \bar{s}^{2}=d r^{2}+e^{2 \psi} \sigma_{i j} d \xi^{i} d \xi^{j}
$$

where $\psi=\psi(r)$, or in conformal coordinates

$$
d \bar{s}^{2}=e^{2 \psi}\left\{d \tau^{2}+\sigma_{i j} d \xi^{i} d \xi^{j}\right\}
$$

where

$$
\tau=\int_{\bar{r}}^{r} e^{-\psi(t)}, \quad 0<\bar{r} \leq r<\frac{\pi}{2},
$$

and $\bar{r}$ very small. Since all hypersurfaces we are concerned with lie in a region

$$
\mathcal{H}\left(x_{0}, r_{0}, r_{1}\right)=\left\{x \in \mathcal{H}\left(x_{0}\right): 0<r_{0} \leq r \leq r_{1}<\frac{\pi}{2}\right\},
$$

in view of Theorem 4.4, choosing $\bar{r}<r_{0}$ ensures that we do not have to worry about a possible coordinate singularity and still have a positive $\tau$-coordinate.

Let $M \subset \mathcal{H}\left(x_{0}, r_{0}, r_{1}\right)$ be a strictly convex hypersurface, then, writing $M$ as a graph

$$
M=\operatorname{graph} u=\left\{(\tau, \xi): \tau=u(\xi), \xi \in S^{n}\right\},
$$

the induced metric and the second fundamental form of $M$ are given by

$$
g_{i j}=e^{2 \psi}\left\{u_{i} u_{j}+\sigma_{i j}\right\}
$$

and

$$
h_{i j} e^{-\psi}=\tilde{h}_{i j}+\psi_{\beta} \tilde{\nu}^{\beta} \tilde{g}_{i j}
$$


where the symbols with the tilde refer to the geometric quantities of $M$, when $M$ is considered to be embedded in the ambient space with metric

$$
d \tilde{s}^{2}=d \tau^{2}+\sigma_{i j} d \xi^{i} d \xi^{j} .
$$

$\tilde{h}_{i j}$ is then given by the relation

$$
v^{-1} \tilde{h}_{i j}=-u_{i j}=-v^{-2} u_{; i j}
$$

where $u_{i j}$ is the Hessian of $u$ with respect to the induced metric $\tilde{g}_{i j}$ and $u_{; i j}$ is the Hessian of $u$ with respect to the standard metric $\sigma_{i j}$ of $S^{n}$. The term $v$ is defined by

$$
v^{2}=1+\sigma^{i j} u_{i} u_{j}
$$

Writing $u_{i j}$ instead of $u_{; i j}$ in the following, we see that

$$
h_{i j} e^{-\psi}=-v^{-1} u_{i j}+v^{-1} \dot{\psi} \tilde{g}_{i j}
$$

where

$$
\dot{\psi}=\frac{d \psi}{d \tau}
$$

If $M$ is invariant under $G$, then the function $u$ is also invariant under the group action. Let $A^{k}(\xi)$ be the local representation of $A \xi$ and $\left(A_{i}^{k}\right)$ its derivative, then the covariant derivatives of $u$ satisfy

$$
u_{i}(\xi)=u_{k}(A \xi) A_{i}^{k}
$$

and

$$
u_{i j}(\xi)=u_{k l}(A \xi) A_{i}^{k} A_{j}^{l}
$$

notice that $A_{i ; j}^{k}=0$.

6.1. Definition. A tensor field $\varphi$ in $T\left(S^{n}\right)$ is called invariant with respect to $G$, if it satisfies transformation relations according to (6.14), (6.15), where the contravariant indices transform like

$$
\varphi^{i}(\xi)=\varphi^{k}(A \xi) A_{k}^{i} \quad \forall A \in G,
$$

and there holds

$$
A_{k}^{i} A_{j}^{k}=\delta_{j}^{i}
$$

These transformation rules hold for invariant tensor fields of arbitrary order.

The metric $\sigma_{i j}$ of $S^{n}$ is of course invariant by the very definition of an isometry. Hence we conclude from (6.12) that the second fundamental form is also invariant and consequently also the tensor

$$
F^{i j}=\frac{\partial F}{\partial h_{i j}}
$$


Now consider the Banach spaces $E_{1}, E_{2}$ defined by

$$
E_{1}=\left\{u \in H^{5, p}\left(S^{n}\right): u \text { invariant }\right\}
$$

and

$$
E_{2}=\left\{w \in H^{3, p}\left(S^{n}\right): w \text { invariant }\right\}
$$

for some fixed $n<p<\infty$, such that $H^{m, p}\left(S^{n}\right) \hookrightarrow C^{m-1, \alpha}\left(S^{n}\right)$.

Let $\Omega \subset E_{1}$ be an open bounded set such that $M(u)=$ graph $u$ is uniformly strictly convex, contained in $\mathcal{H}\left(x_{0}, r_{0}, r_{1}\right)$, such that $x_{0}$ is in interior point of $\widehat{M(u)}$ for all $u \in \Omega$. We then define

$$
\Phi: \Omega \rightarrow E_{2}
$$

by

$$
\Phi(u)=F\left(h_{i j}\right)-f(u, \xi)
$$

expressing a position vector $x \in \mathcal{H}\left(x_{0}\right)$ by $x=(\tau, \xi)$.

All possible solutions of $\Phi=0$ are strictly contained in $\Omega$, if $\Omega$ is specified by the requirements

$$
\begin{gathered}
0<\tau_{0}=\tau\left(r_{0}\right)<u<\tau_{1}=\tau\left(r_{1}\right), \\
x_{0} \in \operatorname{int} \widehat{M(u)},
\end{gathered}
$$

$$
0<\epsilon_{0}<\kappa_{i}<\bar{\kappa}
$$

where $\kappa_{i}$ are the principal curvatures of graph $u$, in view of the a priori estimates in Section 3 and Section 4.

6.2. Lemma. $\Phi$ is a proper nonlinear Fredholm operator of index zero.

Proof. $F$ and hence $\Phi$ are uniformly elliptic in $\Omega$. The properness is due to the a priori estimates in Section 3 and Section 4, the Evans-Krylov and CalderònZygmund estimates and our assumption that $F$ and $f$ are of class $C^{5}$.

If the Banach spaces $E_{i}$ would have been defined without the symmetry requirement, the other properties of $\Phi$ would have been well known. Let $L$ be the derivative of $\Phi$, then $L$ is an elliptic linear partial differential operator of second order

$$
L u=-F^{i j} u_{i j}+b^{i} u_{i}+c u
$$

and the lemma will be proved, if we can show that the operator

$$
-F^{i j} u_{i j}+\lambda u, \quad \lambda>0,
$$

is surjective, i.e., for arbitrary $w \in E_{2}$ there exists $u \in E_{1}$ such that

$$
-F^{i j} u_{i j}+\lambda u=w .
$$


It is well known that there exists a function $u \in H^{5, p}\left(S^{n}\right)$ that solves the preceding equation, and we shall show $u$ is invariant, if $w$ is.

Let $A \in G$, then we claim that $\tilde{u}=u \circ A$ also satisfies (6.28), which would yield

$$
\tilde{u}=u
$$

because of the uniqueness.

Now, differentiating $\tilde{u}=u \circ A$ we obtain

$$
\tilde{u}_{i j}(\xi)=u_{k l}(A \xi) A_{i}^{k} A_{j}^{l}
$$

and we infer

$$
-F^{i j} \tilde{u}_{i j}=-F^{i j} A_{i}^{k} A_{j}^{l} u_{k l}=-F^{k l} u_{k l},
$$

since $F^{i j}$ is invariant.

Recall that $w \in E_{2}$ is said to be a regular value for $\Phi$, if either $w \notin R(\Phi)$, or if for any $u \in \Phi^{-1}(w) D \Phi(u)$ is surjective.

Smale [18] proved that for separable Banach spaces $E_{i}$ and proper Fredholm maps $\Phi$ the set of regular values in $E_{2}$ is open and dense, if $\Phi$ is of class $C^{k}$ such that

$$
k>\max (\operatorname{ind} \Phi, 0) \text {. }
$$

All requirements are satisfied in the present situation.

Next we want to use the uniqueness result in Theorem 5.1. Let $c>0$ be a constant such that

$$
c<\inf _{S^{n}} f
$$

and let $u_{0} \equiv$ const be such that the geodesic sphere $M_{0}=\operatorname{graph} u_{0}$ satisfies

$$
F_{\left.\right|_{M_{0}}}=c .
$$

We assume furthermore that the constants $r_{0}, r_{1}$ and $\epsilon_{0}, \bar{\kappa}$ are chosen such that all possible solutions of

$$
F=t f+(1-t) c, \quad 0 \leq t \leq 1,
$$

in $\mathcal{H}\left(x_{0}\right)$ satisfy the corresponding estimates.

The requirement (6.33) is not essential, it will only simplify some of the following arguments.

Let $0<\delta$ be small and define

$$
\Lambda: \Omega \times[-\delta, 1+\delta] \rightarrow E_{2}
$$


by

$$
\Lambda(u, t)=F\left(h_{i j}\right)-(t f+(1-t) c) .
$$

Then $\Lambda$ is also a proper Fredholm operator such that ind $\Lambda(\cdot, t)=0$ for fixed $t$, and, if $w \in E_{2}$ is a regular value for $\Lambda$, then

$$
\text { ind } \Lambda=1 \quad \forall(u, t) \in \Lambda^{-1}(w) \text {. }
$$

Recall that

$$
\text { ind } \Lambda=\operatorname{dim} N(D \Lambda)-\operatorname{dim} \operatorname{Coker}(D \Lambda) \text {. }
$$

The relation (6.38) will be proved in Lemma 6.5 below.

6.3. Theorem. Let $0<f \in C^{5}\left(S^{n}\right)$ be invariant under $G$, then for any $F \in(K)$ of class $C^{5}$, there exists a strictly convex invariant hypersurface $M \subset \mathcal{H}\left(x_{0}\right)$ satisfying

$$
F_{\left.\right|_{M}}=f
$$

Proof. Consider the Fredholm map $\Lambda=\Lambda(u, t)$. The theorem will be proved, if we can show that there exists $u \in \Omega$ such that

$$
\Lambda(u, 1)=0 \text {. }
$$

On the other hand, there exists a unique solution of the equation

$$
\Lambda(u, 0)=0,
$$

namely, $u=u_{0}$, the geodesic sphere. In the lemma below we shall show that $u_{0}$ is also a regular point for $\Lambda(\cdot, 0)$, or equivalently, $\left(u_{0}, 0\right)$ a regular point for $\Lambda$.

Without loss of generality we may assume $0 \notin R(\Lambda(\cdot, 1))$, for otherwise we have nothing to prove, and thus, 0 is also regular value for $\Lambda(\cdot, 1)$.

Let $\epsilon>0$ be small, then there exists a

$$
w_{\epsilon} \in B_{\epsilon}(0) \subset E_{2},
$$

such that

$$
t f+(1-t) c+w_{\epsilon}>0 \quad \forall-\delta \leq t \leq 1+\delta,
$$

$w_{\epsilon} \in R(\Lambda(\cdot, 0))$, and such that $w_{\epsilon}$ is a regular value for $\Lambda(\cdot, 0), \Lambda(\cdot, 1)$ and $\Lambda$.

Consider

$$
\Gamma_{\epsilon}=\Lambda^{-1}\left(w_{\epsilon}\right) \cap\left(E_{1} \times(-\delta, 1+\delta)\right),
$$

then $\Gamma_{\epsilon} \neq \emptyset$ and $\Gamma_{\epsilon}$ is a 1 -dimensional submanifold without boundary.

The intersection

$$
\tilde{\Gamma}_{\epsilon}=\Gamma_{\epsilon} \cap\left(E_{1} \times[0,1]\right)
$$


is then compact, since $\Lambda$ is proper, and it consists of finitely many closed curves or segments.

We want to prove that there is $u_{\epsilon} \in \Omega$ such that $\left(u_{\epsilon}, 1\right) \in \tilde{\Gamma}_{\epsilon}$. Suppose this were not the case, then consider a point $\left(\bar{u}_{\epsilon}, 0\right) \in \tilde{\Gamma}_{\epsilon}$. Such points exist by assumption. Moreover, the 1-dimensional connected submanifold $M_{\epsilon} \subset \Gamma_{\epsilon}$ containing $\left(\bar{u}_{\epsilon}, 0\right)$ can be expressed near $\left(\bar{u}_{\epsilon}, 0\right)$ by

$$
M_{\epsilon}=\{(\varphi(t), t):-\delta<t<\delta\},
$$

where $\varphi \in C^{1}, \varphi(0)=\bar{u}_{\epsilon}$, and

$$
\Lambda(\varphi(t), t)=w_{\epsilon}
$$

since by assumption $D_{1} \Lambda\left(\bar{u}_{\epsilon}, 0\right)$ is an isomorphism and the implicit function theorem can be applied.

Let $\tilde{M}_{\epsilon}=M_{\epsilon} \cap \tilde{\Gamma}_{\epsilon}$, then $\tilde{M}_{\epsilon}$ isn't closed because of (6.47), and hence has two endpoints, see [12, Appendix]. One of them is $\left(\bar{u}_{\epsilon}, 0\right)$ and the other also belongs to $\Lambda(\cdot, 0)^{-1}\left(w_{\epsilon}\right)$ and can therefore be expressed as

$$
\left(\tilde{u}_{\epsilon}, 0\right)
$$

where $\tilde{u}_{\epsilon} \neq \bar{u}_{\epsilon}$ because of the implicit function theorem.

Hence we have proved that the assumption

$$
\Lambda(\cdot, 1)^{-1}\left(w_{\epsilon}\right)=\emptyset
$$

implies

$$
\# \Lambda(\cdot, 0)^{-1}\left(w_{\epsilon}\right) \text { is even. }
$$

However, we shall show that $\Lambda(\cdot, 0)^{-1}\left(w_{\epsilon}\right)$ contains only one point, if $\epsilon$ is small.

Indeed, let $\bar{u}_{\epsilon} \in \Lambda(\cdot, 0)^{-1}\left(w_{\epsilon}\right)$, then the $\bar{u}_{\epsilon}$ converge to the unique solution $u_{0}$ of (6.42). Thus, if $\epsilon$ is small all $\bar{u}_{\epsilon}$ are contained in an open ball

$$
B_{\rho}\left(u_{0}\right) \subset \Omega,
$$

where $\Phi=\Lambda(\cdot, 0)$ is a diffeomorphism due to Lemma 6.4 , hence there exists just one solution of the equation

$$
\Lambda\left(\bar{u}_{\epsilon}, 0\right)=w_{\epsilon} .
$$

Thus we have proved that there exists a sequence

$$
u_{\epsilon} \in \Lambda(\cdot, 1)^{-1}\left(w_{\epsilon}\right),
$$

if $\epsilon$ tends to zero. A subsequence will then converge to a solution $u$ of

$$
\Lambda(u, 1)=0 \text {. }
$$

It remains to prove two lemmata

6.4. Lemma. $u_{0}$ is a regular point for $\Lambda(\cdot, 0)$. 
Proof. Let $\varphi \in E_{1}$ be arbitrary and $\epsilon>0$ so small that

$$
u=u_{0}+\epsilon \varphi \in \Omega \text {. }
$$

Then we have to calculate

$$
\frac{d}{d \epsilon} \Lambda(u, 0)_{\left.\right|_{\epsilon=0}}=\frac{d}{d \epsilon}\left\{F\left(h_{i j}\right)-c\right\} .
$$

Now,

$$
\frac{d}{d \epsilon} F\left(h_{i j}\right)=F_{j}^{i} \dot{h}_{i}^{j}
$$

and

$$
h_{i}^{j}=-v^{-1} e^{-\psi} \tilde{g}^{j k} u_{i k}+\dot{\psi} e^{-\psi} \delta_{i}^{j},
$$

in view of (6.12), where

$$
\tilde{g}^{j k}=\sigma^{j k}-\frac{u^{j} u^{k}}{v^{2}} .
$$

Evaluating the resulting expressions at $\epsilon=0$ we conclude

$$
\dot{h}_{i}^{j}=-e^{-\psi} \varphi_{i}^{j}+\left\{\ddot{\psi}-|\dot{\psi}|^{2}\right\} e^{-\psi} \delta_{i}^{j} \varphi
$$

hence,

$$
\frac{d}{d \epsilon} F\left(h_{i j}\right)=e^{-\psi}\left\{-\Delta \varphi-n\left(|\dot{\psi}|^{2}-\ddot{\psi}\right) \varphi\right\},
$$

where the Laplace operator is taken with respect to the metric in $S^{n}$ and $e^{-\psi}$ is a constant.

Looking at the equations (4.10), (4.11) we deduce that $\psi$ can be expressed as a function of $\tau$ as

$$
\psi=\log \rho-\log \left(1+\frac{1}{4} \rho^{2}\right), \quad \rho=e^{\tau+\tau_{0}},
$$

where $\tau_{0}$ is an integration constant depending on the value of $\bar{r}$ in (6.4), yielding

$$
|\dot{\psi}|^{2}-\ddot{\psi}=1 \text {. }
$$

Thus $\varphi \in N\left(D_{1} \Lambda\left(u_{0}, 0\right)\right)$ satisfies

$$
-\Delta \varphi-n \varphi=0
$$

and is therefore a spherical harmonic of degree 1 or identically zero. But the $G$ invariant functions are orthogonal to the spherical harmonics of degree 1 , hence $D_{1} \Lambda\left(u_{0}, 0\right)$ is an isomorphism.

6.5. Lemma. Let $\Lambda$ be defined as above, then

$$
\text { ind } \Lambda=1 \text {. }
$$


Proof. Let $\left(u_{0}, t_{0}\right) \in E_{1} \times[-\delta, 1+\delta]$ be fixed, where we may assume that $t_{0}=1$, since $\Lambda$ is continuous. We distinguish two cases:

Case 1: $\quad(f-c) \in R\left(D \Phi\left(u_{0}\right)\right)$

Notice that $\Lambda$ can be extended as a class $C^{2}$-function to $E_{1} \times \mathbb{R}$. We have

$$
D \Lambda=\left(D_{1} \Lambda,-(f-c)\right)
$$

where all derivatives are evaluated at $\left(u_{0}, 1\right)$ resp. $u_{0}$. Then we deduce

$$
\operatorname{dim} N(D \Lambda)=\operatorname{dim} N\left(D_{1} \Lambda\right)+1=\operatorname{dim} N(D \Phi)+1,
$$

for let

$$
D_{1} \Lambda u_{1}=f-c
$$

then

$$
N(D \Lambda)=N(D \Phi) \times\{0\} \oplus\left\langle\left(u_{1}, 1\right)\right\rangle
$$

as one easily checks, and of course there holds

$$
R(D \Lambda)=R(D \Phi)
$$

Case 2: $\quad(f-c) \notin R\left(D \Phi\left(u_{0}\right)\right)$

In this case

$$
R(D \Lambda)=R\left(D_{1} \Lambda\right) \oplus\langle(f-c)\rangle
$$

and

$$
N(D \Lambda)=N\left(D_{1} \Lambda\right) \times\{0\},
$$

hence

$$
\text { ind } \Lambda=\operatorname{ind} \Phi+1=1
$$

in both cases.

\section{Proof of Theorem 0.4}

The barrier condition for the original pair $(F, f)$ in $\mathcal{H}\left(-x_{0}\right)$ immediately translates to a barrier condition for $\left(\tilde{F}, f^{-1}\right)$ in $\mathcal{H}\left(x_{0}\right)$. Following the stipulations in Remark 0.5, we again assume that we consider the problem

$$
F_{\left.\right|_{M}}=f(x) \quad \forall x \in M,
$$

where $F \in(K)$ and $M_{1}$ resp. $M_{2}$ are lower resp. upper barriers for $(F, f)$ bounding a connected open set $\Omega \subset \mathcal{H}\left(x_{0}\right)$.

We want to apply an old result, [7, Theorem 0.4], in which we showed that the problem (7.1) has a strictly convex solution $M \subset \bar{\Omega}$ of class $C^{6, \alpha}$ assuming 
that $F$ is of class $(K)$, homogeneous of degree 1 , and concave. In addition there should exist a strictly convex function $\psi \in C^{2}(\bar{\Omega})$. The ambient space was an arbitrary Riemannian manifold $N, \bar{\Omega}$ was supposed to be compact, and should be covered by a normal Gaussian coordinate system $\left(x^{\alpha}\right)$.

All hypotheses are satisfied in the present situation: $\bar{\Omega}$ is compact, the normal Gaussian coordinate system is given by choosing geodesic polar coordinates with center in $x_{0}$, the strictly convex function $\psi$ can be defined by

$$
\psi=\frac{1}{2}\left|x^{0}\right|^{2}
$$

where $x^{0}$ is the radial distance to $x_{0}$, as one easily checks observing that the level hypersurfaces $\left\{x^{0}=\right.$ const $\}$ which intersect $\bar{\Omega}$ are all uniformly strictly convex, and $F$ is homogeneous of degree 1 and therefore also concave.

\section{REFERENCES}

[1] A.D. Alexandrov, On the theory of mixed volumes of convex bodies, Mat. Sb. 3 (1938), $27-46$.

[2] Shiu Yuen Cheng and Shing Tung Yau, On the regularity of the solution of the $n$-dimensional Minkowski problem, Comm. Pure Appl. Math. 29 (1976), no. 5, 495-516.

[3] M. P. do Carmo and F. W. Warner, Rigidity and convexity of hypersurfaces in spheres, J. Differential Geometry 4 (1970), 133-144.

[4] Lawrence C. Evans, Classical solutions of fully nonlinear, convex, second-order elliptic equations, Comm. Pure Appl. Math. 35 (1982), no. 3, 333-363.

[5] Claus Gerhardt, Closed Weingarten hypersurfaces in Riemannian manifolds, J. Diff. Geom. 43 (1996), 612-641, pdf file.

[6] Closed Weingarten hypersurfaces in space forms, Geometric Analysis and the Calculus of Variations (J Jost, ed.), International Press, Boston, 1996, pdf file, p. 71-98.

[7] — Hypersurfaces of prescribed Weingarten curvature, Math. Z. 224 (1997), 167-194, download pdf file.

[8] - Hypersurfaces of prescribed curvature in Lorentzian manifolds, Indiana Univ. Math. J. 49 (2000), 1125-1153, arXiv:math.DG/0409457.

[9] Bo Guan and Pengfei Guan, Convex hypersurfaces of prescribed curvatures., Ann. Math. 156 (2002), 655-673.

[10] N. V. Krylov, Nonlinear elliptic and parabolic equations of the second order, Mathematics and its Applications (Soviet Series), vol. 7, D. Reidel Publishing Co., Dordrecht, 1987.

[11] Hans Lewy, On differential geometry in the large. I. Minkowski's problem, Trans. Amer. Math. Soc. 43 (1938), no. 2, 258-270.

[12] John W. Milnor, Topology from the differentiable viewpoint, Based on notes by David W. Weaver, The University Press of Virginia, Charlottesville, Va., 1965.

[13] H. Minkowski, Volumen und Oberfläche., Math. Annalen 43 (1903), 447-495.

[14] Louis Nirenberg, The Weyl and Minkowski problems in differential geometry in the large, Comm. Pure Appl. Math. 6 (1953), 337-394.

[15] A. V. Pogorelov, Regularity of a convex surface with given Gaussian curvature, Mat. Sbornik N.S. 31(73) (1952), 88-103.

[16] Rolf Schneider, Closed convex hypersurfaces with curvature restrictions, Proc. Amer. Math. Soc. 103 (1988), no. 4, 1201-1204.

[17] Convex bodies: the Brunn-Minkowski theory, Encyclopedia of Mathematics and its Applications, vol. 44, Cambridge University Press, Cambridge, 1993. 
[18] S. Smale, An infinite dimensional version of Sard's theorem, Amer. J. Math. 87 (1965), 861-866.

[19] Michael Spivak, A comprehensive introduction to differential geometry. Vol. I-V. 2nd ed., Publish Perish, Inc., Berkeley, 1979.

Claus Gerhardt

Ruprecht-Karls-Universität, Institut für Angewandte Mathematik

Im Neuenheimer Feld 294, 69120 Heidelberg, Germany

E-mail: gerhardt@math.uni-heidelberg.de

URL: http://www.math.uni-heidelberg.de/studinfo/gerhardt/ 